\title{
BIOTECNOLOGÍA EN LA INDUSTRIA DE LA PULPA, Y EL PAPEL: MAPEO DE PATENTES
}

Laura Emila Cerón Rincón*

\section{RESUMEN}

La biotecnología se plantea como una oportunidad para los países en desarrollo, Colombia cuenta con condiciones que favorecen el desarrollo de esta área, como una fuerte tradición en infraestructura básica de investigación principalmente en los campos de agricultura y la salud humana. Las iniciativas regionales han ayudado a levantar el perfil de la industria biotecnológica como: $E l$ Cluster de Bioindustrial del Valle del Cauca, El ejercicio Prospectivo en Biotecnología y Salud de Antioquia y La iniciativa de Bioprogreso en la región Bogotá-Cundinamarca. Según la Cámara de Comercio de Bogotá (2005) se consideran a la cadena productiva del papel y artes gráficas como estratégicas para la región de Bogotá D.C.Según la visión de la Agenda Interna para la Productividad y la Competitividad del Departamento Nacional de Planeación (2007) para dicha cadena, esta alcanzará progresivamente mayor competitividad y mayor inserción en el mercado internacional globalizado, llegando a triplicar sus exportaciones para el año 2015, entre las acciones identificadas para lograr este objetivo el se encuentra el Desarrollo tecnológico, donde se propone aprovechar los avances en biotecnología. Dado esto, se recopilaron patentes que incluyeran desarrollos biotecnológicos para la industria de la pulpa y el papel del año 2000 hasta la fecha, a partir de las cuales se realizo un análisis de la información recopilada a través de una herramienta de vigilancia tecnológica con la que generó un mapa tecnológico, como un primer paso de un proceso dirigido a contribuir en la definición de políticas y direccionamiento de la gestión tecnológica para que la cadena de papel.

\section{Palabras clave}

Biotecnología, industria del papel y la pulpa, mapeo tecnológico.

\section{InTroducción}

El uso y desarrollo de la biotecnología para la generación de bienes y servicios en el país tiene como fin contribuir a mejorar la competitividad y ampliar los mercados de la producción nacional (Colciencias,2008; Conpes 3527),promover esta área en el país en la generación de bienes y servicios, contribuirá a mejorar la competitividad y ampliar los mercados de la producción nacional (Colciencias, 2008), su potencial de generar nuevos productos $y$ procesos, necesariamente debe responder a las necesidades del sector productivo. La biotecnología se ha definido como un área estratégica para los países en desarrollo entre ellos América latina y Colombia cuenta con una serie de condiciones que favorecen el desarrollo de esta área, como una infraestructura básica de investigación e integración de diferentes conocimientos como la biología, la medicina, la agricultura, así como una abundante disponibilidad de biodiversidad y recursos

\footnotetext{
Química, MSc Microbilogía. cPhD, Biotecnología.
} 
genéticos. La Biodiversidad uno de los pilares para el desarrollo del área se encuentra amenazada por el impacto de las actividades humanas, afrontar esta amenaza requiere por una parte comprender cómo las actividades humanas están afectando el medio ambiente y por otra, plantear alternativas de uso de los recursos naturales que mantengan tanto la calidad ambiental, como la eficiencia de la producción que a partir de ellos se obtiene es decir, alternativas sostenibles. Llevar a cabo este ideal requiere de la búsqueda sistemática en la biodiversidad de: genes, compuestos, macro y microorganismos con potencial uso biotecnológico (bioprospección), etapa de investigación que debe contar con grupos o instituciones científicas que estén en capacidad de otorgar valor agregado a los procesos biológicos, a través de su aplicación industrial, propendiendo por su conservación y por el desarrollo económico de los países. El valor que tienen sus recursos naturales no renovables y la biodiversidad esta dado en gran medida por el desarrollo de nuevos bioproductos e industrias usando los recursos genéticos de la flora y fauna, de microorganismos y de otros recursos biológicos, en esta medida el aprovechamiento de la biodiversidad mediante la biotecnología. Para el desarrollo y la competitividad de esta área estratégica requiere condiciones fundamentales como la participación activa de todos los actores involucrados en la toma de decisiones sobre el desarrollo de la biotecnología y sus aplicaciones, así como su articulación con las políticas de otros sectores de la economía nacional.

\section{Abstract}

This paper provides an explanation on why the Health cluster in Medellin may turn into an alternative competitive advantage of great added value for the city. This aims at the possibility of exporting medical services characterized by a vanguard spirit in research and the development of specialized medical procedures.

The high health level reached, together with the proper consolidation of the educational, industrial, commercial, and service sectors of the city and other places in the region of Antioquia,

complement the development of the initiative aiming at generating differential issues in the field of socio- economic competitiveness. Currently, the strategic cluster of Medellin city and the region of Antioquial wants to develop five economic sectors that are described above. For this reason, the authors show how the health cluster has the major competitive potential compared with the other four sectors.

\section{KEY WORDS}

Cluster

Health

Services 
La Cámara de Comercio de Bogotá (2005) ha caracterizado sectores estratégicos para la región dentro de las cadenas productivas de manufactura y servicios en Bogotá y Cundinamarca, resaltando como cadenas estratégicas para la región la de químicos y petroquímicos y papel y artes gráficas. Según la visión de la Agenda Interna para la Productividad y la Competitividad del Departamento Nacional de Planeación (2007) la cadena de pulpa, papel, cartón, industria gráfica, editoriales y empresas conexas, alcanzará progresivamente mayor competitividad y mayor inserción en el mercado internacional globalizado, llegando a triplicar sus exportaciones para el año 2015. Además, logrará operaciones rentables, sostenibles y con sentido de responsabilidad ambiental y social, entre las acciones identificadas para lograr este objetivo el se encuentra el Desarrollo tecnológico, donde se propone que los avances en la biotecnología.

\section{Marco ReFERENCIAL}

\section{Ua biotecnología en Colom-} bia. Como marco referencial del estado actual de la biotecnología en Colombia aplicable al contexto empresarial, se puede decir que en 1991 con el Programa Nacional de Biotecnología se crea un instrumento para el fomento de la capacidad científica y promoción del desarrollo del área, en el plan estratégico 1999-2004 se definen las líneas de acción. Actualmente en el país se cuenta con una importante tradición e infraestructura de investigación principalmente en los campos de agricultura y la salud humana, existen 134 grupos de investigación en biotecnología (Roca,2004) y de acuerdo con el sector de aplicación están divididos en $58 \%$ en el sector vegetal y agrícola, $17 \%$ en salud humana, $12 \%$ en ambiental, $8 \%$ en pecuario y $5 \%$ en industrial. Representados por Instituciones y empresas como El Instituto de Biotecnología IBUN, Facultad de Ciencias y Facultad de Agronomía de la Universidad Nacional, Corpoica, Centro Internacional de Agricultura Tropical CIAT, Servicio Nacional de Aprendizaje SENA, CorpoGen, Centro de Investigación y Desarrollo Tecnológico de Compañía Colombiana de Tabaco S.A., Corporación para Investigaciones Biológicas $\mathrm{CIB}$, Vecol S.A., Centro de investigaciones microbiológicas y Departamento de Ingeniería Química de la Universidad de los Andes, Life Systems Technology S.A. (LST), Laverlam S.A., Grupo de salud animal de la Universidad de Antioquia. El Instituto Alexander Von Humboldt, Corporación Biotec, Corporación para el Desarrollo Industrial de la Biotecnología y Producción Limpia CORPODIB, entre las mas importantes.

Las iniciativas regionales han ayudado a levantar el perfil de la industria como el Cluster de Bioindustrial del Valle del Cauca dado el potencial existente en el occidente colombiano en cuanto a los recursos biológicos y tejido 
empresarial e institucional, mediante el dimensionamiento de 10 cadenas productivas bioindustriales. Se resalta la clara articulación que existe entre las cadenas productivas bioindustriales identificadas en la Estrategia Bioregión Valle del Cauca 2019 y las apuestas productivas consideradas en la Agenda Interna para la Productividad y Competitividad del Departamento (2005), particularmente en: la industria agroalimentaria y agropecuaria, caña de azúcar, forestales y maderables, industria papelera y derivados, producción pesquera y aprovechamiento marino, productos naturales derivados del uso sostenible de la biodiversidad, biomédica y software para la bioindustria. El ejercicio prospectivo en Biotecnología y Salud de Antioquia, reúne 112 proyectos en el marco de la ciencia, la tecnología y la innovación en los siguientes sectores: Agroindustria e industria de alimentos, Salud y ciencias de la vida, Fibras, textiles y confecciones, Servicios de ingeniería y consultoría, Agua y medio ambiente.

La iniciativa de Bioprogreso en la región Bogotá-Cundinamarca reúne un gremio de cinco empresas de biotecnología bogotanas, que junto con la Cámara de Comercio de Bogotá trabaja para mejorar la cadena Hortofruticula enmarcada dentro Mega Proyecto agroindustrial de la sabana Cundiboyacense (Cámara de Comercio de Bogotá, 2006).

\section{Biotecnología en industria} del papel y la pulpa. Como ya se mencionó la Cámara de Comercio de Bogotá (2005) ha caracterizado sectores estratégicos para la región dentro de las cadenas productivas de manufactura y servicios en Bogotá y Cundinamarca, resaltando como cadenas estratégicas para la región la de químicos y petroquímicos y papel y artes gráficas. La implementación de procesos amigables con el ambiente se ha convertido en una meta para la industria del papel y la pulpa, donde la investigación en biotecnología ha tenido un gran impacto en la última década, particularmente hacia el uso de microorganismos y sus enzimas capaces de descomponer materiales lignocelulolíticos como es el caso de los hongos (Pycnoporus cinnabarinus, Trametes versicolor $y$ Pleurotus eryngii) de la pudrición blanca de la madera, para ser aplicados tanto en procesos de producción de pulpa como en le blanqueado, sin embargo además hay que tener en cuenta que esta tecnología debe estar acompañada de bioactivadores y el estudio de los subproductos resultantes.

$$
\text { El propósito es reducir o }
$$
reemplazar la extracción alcalina de la hemicelulosa y los requerimientos de cloro en los procesos de blanqueado, sin afectar las fibras de celulosa y así mantener las cualidades y calidad de los productos de papel, adicionalmente los residuos presentes en los efluentes puede ser bio convertirlos a productos como el etanol (Sapre et al, 2005). En la manufactura de pulpas de papel de 
gran valor los procesos de blanqueado del papel utilizan productos que contienen cloro, es factible sustituir estos productos por el blanqueado enzimático, tecnología que se basa en la utilización de la actividad laccasa, enzima que puede ser producida por los hongos mencionados anteriormente y que está involucrada en la degradación de lignina a través de la oxidación de compuestos fenólicos, además se utiliza en la mineralización de un amplio rango de colorantes sintéticos. Dado lo anterior se aplica en varios procesos como la obtención de biopulpa, bioblanqueado, biorremediacion, biosensores, varios usos en tecnología de alimentos y en el tratamiento de aguas residuales industriales (Hao et al, 2007).

Se define el proceso de obtención de biopulpa (Biopulping) como el tratamiento de trozos de madera con hogos degradadores de lignina antes de obtener la pulpa. El pre tratamiento fúngico anterior a tratamientos mecánicos de obtención de pulpa reduce los requerimientos de energía eléctrica durante el refinado o bien aumenta la cochada para el molino, aumenta la fuerza del papel, reduce el contenido de resinas y reduce el impacto ambiental del procesos de obtención de la pulpa. La enzima xilanasa en cuya producción industrial se utiliza principalmente el hongo Trichoderma sp., cataliza la hidrolisis del xilano polisacárido que constituye principalmente junto con la celulosa la hemicelulosa de la pared vegetal, esta enzima se utiliza extensivamente en los procesos de alimentos y en la industria química y de pulpa, en procesos de obtención de biopulpa y de bioblanqueado, ya que provee ventajas como descenso en el consumo de cloro, la demanda química de oxigeno, con el consecuente incremento de la calidad del agua residual proveniente del proceso, (Kar et al., 2006).

Los procesos de innovación y desarrollo de las nuevas tecnologías requieren de la aplicación de nuevas herramientas para la asimilación de la variable tecnológica en los sistemas productivos, así como la dinamización en la toma de decisiones y la permanente definición de estrategias tecnológicas (Castellanos et al., 2006). Para el caso de la innovación en la industria de la pulpa y el papel, se realizó un mapeo de patentes que incluyeran desarrollos biotecnológicos, dado que en las patentes se encuentran las tecnologías que están próximas a salir al mercado proporcionando una visión del desarrollo tecnológico, se utilizo una metodología de vigilancia tecnológica que se basa en la transformación de la información contenida en bases de datos, mediante la elaboración de mapas tecnológicos, como parte de los pasos que conllevan a realizar procesos de Vigilancia Tecnológica en un contexto de interés.

\section{El proceso de la vigilancia}

tecnológica. Consta de la búsqueda de información, del análisis de datos manteniendo un balance entre calidad y cantidad y estableciendo la interacción entre estos, para generar información 
que desemboque en un conocimiento útil a los procesos productivos y posteriormente en un nuevo desarrollo (producto). En el entorno actual de desarrollo continuo y acelerado, se ha creado la necesidad de incorporar en el componente productivo distintos elementos diferenciadores que permitan anticipar los cambios tecnológicos. En este contexto, la vigilancia tecnológica (VT) surge como una metodología enfocada a analizar estos cambios y a identificar retos y oportunidades, apoyándose principalmente en las tecnologías de información ( $\mathrm{TI}$ ), mediante la búsqueda, captura y análisis de datos e información (León A, 2006).

Dentro de la VT se hace necesario generar criterios para la escogencia y la utilización eficientes de las herramientas de software con distintas características, requerimientos, capacidades y costos, que pueden ser utilizados en dicho proceso. Inicialmente se realiza una aproximación a los distintos modelos desarrollados en VT, haciendo énfasis en la identificación y análisis de las diversas fuentes de información, por su cobertura, aporte al proceso de vigilancia, tipo de insumos informático y acceso; posteriormente se proponen algunos criterios para la valoración, selección y análisis de pertinencia por contexto, de acuerdo al perfil y necesidad individual de quienes realizan VT, para el uso de este tipo de herramientas y finalmente se describen algunos de los paquetes de software existentes en el mercado para la realización de proyectos de vigilancia, relacionándolos con su complejidad, sus características de proceso y sus costos.

La vigilancia, tecnológica(VT) involucra procesos de planeación, dirección, control y coordinación del desarrollo e implementación de la información, para entender y anticiparse a los cambios tecnológicos, haciendo una detección temprana de eventos que representan oportunidades o amenazas potenciales.

De esta manera, la VT, implica un trabajo importante de análisis, en términos de definir los avances en las distintas áreas tecnológicas, difundirlos a la gente correcta y apoyar a la toma de decisiones estratégicas (Liao, 2005; Rodríguez, 2003).

A su vez la VT, requiere de esquemas de búsqueda de información que permitan abordar de manera eficiente la consecución de información del entorno y traducirlo en conocimiento útil para solventar las necesidades $\mathrm{y}$ retos del desarrollo tecnológico, los cuales no siempre son correctamente identificados y adecuadamente implementados en modelos de gestión, conduciendo generalmente a una ausencia en los resultados efectivos.

Identificar y definir una necesidad real y clara de información, representa uno de los mayores desafíos en la priorización de la vigilancia, en el ámbito tecnológico. De manera análoga a un sistema o modelo de computación, en la cual la calidad de los resultados depende en gran medida del insumo con 
el que son alimentados, la validez de los resultados de la vigilancia tecnológica dependerá de las fuentes de información, sus procesos y herramientas de análisis, así como de la competencia del equipo de profesionales responsables del proyecto. Por ello, es relevante tener fuentes de información, confiables y adecuadas para soportar eficientemente cada fase de la vigilancia y de esta manera reducir el tiempo de análisis y tomar de decisiones acertadas.

\section{Metodología}

Para la generación del mapeo tecnológico se desarrollo el método descrito por Vargas y Castellanos (2005) como se describe a continuación: Se realizo una búsqueda de patentes que incluyeran desarrollos biotecnológicos para la industria de la pulpa y el papel del año 2000 hasta la fecha, las búsquedas se hicieron en fuentes primarias como bases de datos disponibles por vía web: http://www. freepatentsonline.com/ y http://www. google.com/patents.
Teniendo en cuenta términos técnicos relacionados con el tema de interés como fueron: paper pulp bleaching, laccase, polyphenoloxidase, enzymatic bleaching, pulp bleaching, lignocellulosic material bleaching, pulp delignification, delignification, depolymerizing lignin, lignin degradation, biodegradation of lignin, pulp or paper manufacturing process, lignolytical enzymes, ligninase, peroxidase, lignin peroxidase, xylanase, biopulping, biobleaching, enzyme mixtures, entre otras.

De los registros encontrados (alrededor de 1500) se descartaron los desarrollos relacionados con biorremediación de compuestos fenólicos y halogenados, tratamiento de efluentes y procesos de conversión de azucares para la producción de etanol. Seleccionando las patentes aplicables al los procesos de blanqueo y/o deslignificación en la manufactura del papel. Las patentes seleccionas se caracterizaron por palabras clave que fueron extraídas de cada patente después de analizar su titulo y resumen, para evitar ambigüedad en la caracterización. Los datos se organizaron en una hoja de calculo en Microsoft $₫$ Excel 2007, como se ilustra en la figura 1. 


\begin{tabular}{|c|c|c|c|c|c|}
\hline PATENTE & AÑo & & & PALABRAS CLAVE & \\
\hline US $7,320,741$ B2 & 2008 & Xylanases & chlorine dioxide bleaching & pulp bleaching & \\
\hline US 7,368,036 B2 & 2008 & Xylanases & chlorine dioxide bleaching & hydrogen peroxide & pulp bleaching \\
\hline US 7,294,228 B2 & 2007 & microorganism & biopulping & & \\
\hline US 7,022,511 B2 & 2006 & Lignin degradation & Consortium of Bacteria & Isolation of bacteria & Acclimatation of bacteria \\
\hline US $7,018,510 \mathrm{~B} 2$ & 2006 & Lignin degradation & biopulping & Consortium of Bacteria & \\
\hline US 7,138,035 B2 & 2006 & Peroxidase & selective oxidation & Carbohydrates & environmentally friendly \\
\hline US $7,008,505$ B2 & 2006 & biopulping & lignin degradation & white-rot fungi & wood waste \\
\hline US 7,125,471 B2 & 2006 & Enzyme mixtures & Manufacture of paper & sludge & \\
\hline US $6,958,110$ B2 & 2005 & Enzyme mixtures & microorganism & Manufacture of paper & lignin degradation \\
\hline US 6,939,437 B1 & 2005 & cellulolitic enzymes & Manufacture of paper & cationic polymer & \\
\hline $\begin{array}{l}\text { US } 6,824,646 \text { B2 } \\
\text { US } 6,767,728 \text { B2 }\end{array}$ & $\begin{array}{l}2004 \\
2004\end{array}$ & $\begin{array}{r}\text { cellulolitic enzymes } \\
\text { Enzyme mixtures }\end{array}$ & $\begin{array}{l}\text { Lignin degradation } \\
\text { b-glucosidase }\end{array}$ & $\begin{array}{l}\text { pulp bleaching } \\
\text { waste paper }\end{array}$ & $\begin{array}{r}\text { reverse osmosys } \\
\text { deinking }\end{array}$ \\
\hline US 6,506,593 B2 & 2003 & Xylanases & Bacterial protein & Lignin degradation & Recombinant technology \\
\hline US $6,613,192$ B1 & 2003 & biopulping & Manufacture of paper & Lignin degradation & white-rot fungi \\
\hline US $6,610,172$ B1 & 2003 & Laccase & Manufacture of paper & paper wet strength & \\
\hline US $6,660,128$ B1 & 2003 & Laccase & peroxidase & biopulping & lignin degradation \\
\hline US 6,387,211 B1 & 2002 & Oxidoreductase & Reducing fluorescence & Manufacture of paper & \\
\hline US $6,402,887$ B1 & 2002 & white-rot fungi & biopulping & Manufacture of paper & Lignin degradation \\
\hline $\begin{array}{l}\text { US } 6,465,203 \text { B2 } \\
\text { US } 6,379,948 \text { B1 }\end{array}$ & $\begin{array}{l}2002 \\
2002\end{array}$ & $\begin{array}{r}\text { glucosyltransferase } \\
\text { microorganism }\end{array}$ & $\begin{array}{l}\text { glucans production } \\
\text { lignin degradation }\end{array}$ & Manufacture of paper & \\
\hline US $6,426,200$ B2 & 2002 & Enzyme mixtures & b-glucosidase & laser printed paper & deinking \\
\hline US $6,468,391$ B1 & 2002 & cellulolitic enzymes & Manufacture of paper & & \\
\hline US $6,242,245$ & 2001 & oxidoreductase & Lignin degradation & Aditive & \\
\hline US 6,284,479 B1 & 2001 & glucosyltransferase & Modified starch & Manufacture of paper & Streptococcus mutans \\
\hline US $6,258,209$ B1 & 2001 & Enzyme mixtures & lignin degradation & & \\
\hline US 6,099,688 & 2000 & cellulolitic enzymes & biopulping & Trichoderma & Aspergillus/Penicillum/Streptomyces \\
\hline US $6,066,233$ & 2000 & Enzyme mixtures & cellulolitic enzymes & pectinase & biopulping \\
\hline US $6,027,610$ & 2000 & Enzyme mixtures & biopulping & cellulolitic enzymes & lipase \\
\hline US $6,057,438$ & 2000 & Xylanases & Cellulosic material & & \\
\hline
\end{tabular}

De las 44 palabras clave con las que fueron caracterizadas las patentes, se escogieron 18 por que aparecen en más de un documento, la frecuencia de aparición de las palabras seleccionadas se ilustra en la figura 2.

Con las 18 palabras seleccionadas se genero una matriz de ocurrencia y coocurrencia de las palabras clave, donde las palabras se disponen como filas y columnas, esta matriz muestra la frecuencia de aparición de una palabra con otra en una misma patente, la diagonal de esta matriz simétrica muestra la frecuencia de aparición de una palabra clave, la matriz se normalizo utilizando el índice de Jaccar, que corresponde a un índice cienciométrico de similaridad (Ivchenko y Honov, 1998). Este índice es la razón entre el número de documentos que tienen dos palabras clave y la suma total de los documentos en que se encuentran las dos palabras, como se ilustra en la fórmula 1.

\section{Fórmula 1: Indice de Jaccar}

$J(i j)=C i j / C i+C j-C i j$

$\mathrm{Cij}=$ número de ocurrencias de la palabra i e j

$\mathrm{Ci}=$ frecuencia de la palabra $\mathrm{i}$

$\mathrm{Cj}=$ frecuencia de la palabra $\mathrm{j}$

La normalización arroja valores entre 0 y 1 . Corresponde al valor uno (1) cuando dos palabras aparecen solamente en los mismos documentos $(\mathrm{C} \mathrm{i}=\mathrm{Cj}=\mathrm{C} \mathrm{ij})$ y corresponda al valor cero (0) cuando las dos palabras no aparecen citadas en forma conjunta en ningún documento $(\mathrm{Cij}=0)$. Parte de la matriz obtenida por este proceso se ilustra en la figura 3. 
Figura 2

Frecuencia de las palabras clave

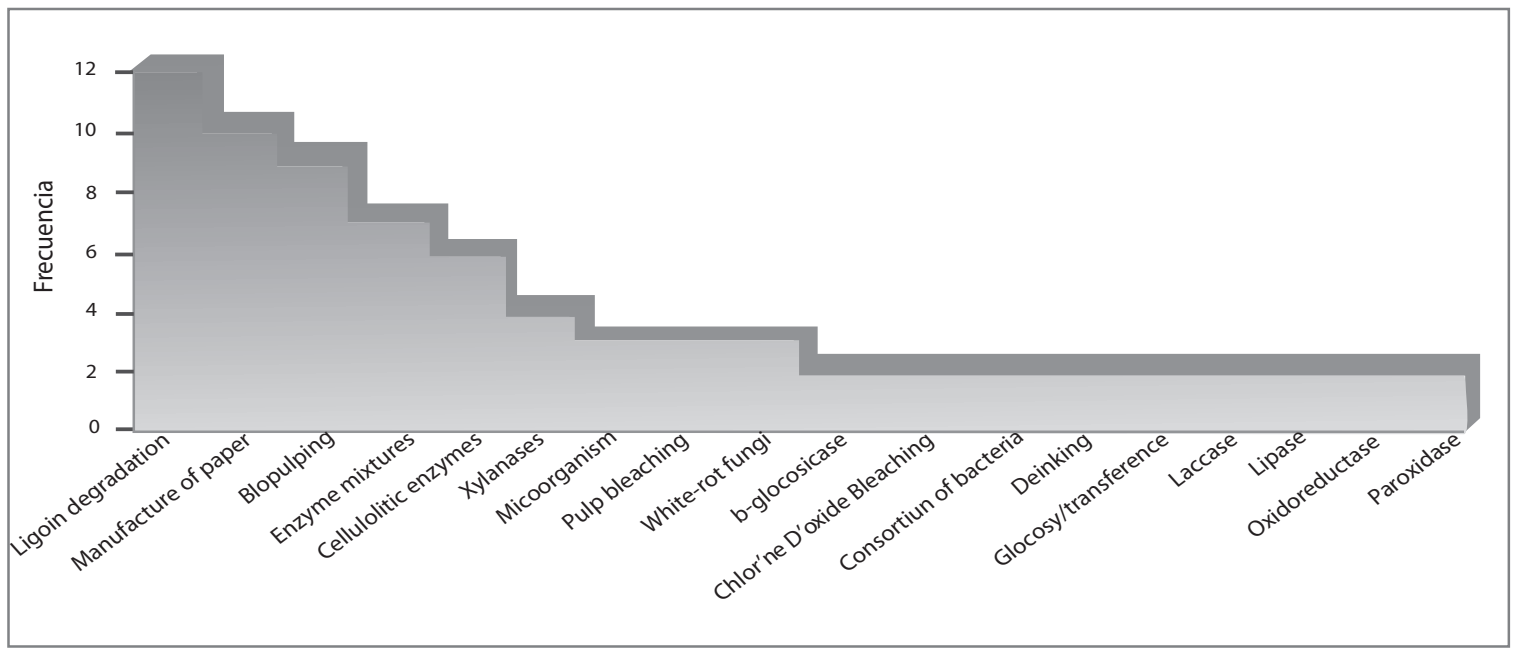

Figura 3

Matriz normalizada de ocurrencia y coocurrencia de palabras claves en las patentes

\begin{tabular}{|c|c|c|c|c|c|c|c|c|c|c|c|c|}
\hline \multirow[b]{2}{*}{ Lignin Degradation } & \multicolumn{6}{|c|}{ 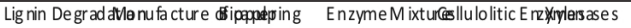 } & \multicolumn{6}{|c|}{ 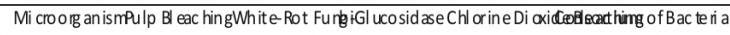 } \\
\hline & 1,00 & 0,16 & 0,36 & 0,07 & 0,06 & 0,07 & 0,07 & 0,07 & 0,15 & 0,17 & 0,00 & 0,18 \\
\hline Manufacture of paper & 0,16 & 1,00 & 0,12 & 0,06 & 0,25 & 0,00 & 0,08 & 0,00 & 0,18 & 0,00 & 0,00 & 0,00 \\
\hline Biopulping & 0,36 & 0,12 & $1, \infty$ & 0,07 & 0,15 & 0,00 & 0,09 & 0,00 & 0,33 & 0,00 & 0,00 & 0,10 \\
\hline Enzyme Mixtures & 0,07 & 0,06 & 0,07 & 1,00 & 0,08 & 0,00 & 0,11 & 0,00 & 0,00 & 0,00 & 0,00 & 0,00 \\
\hline Cellulo litic Erzymes & 0,06 & 0,25 & 0,15 & 0,08 & 1,00 & 0,00 & 0,00 & 0,13 & 0,00 & 0,00 & 0,00 & 0,00 \\
\hline Xylanases & 0,07 & 0,00 & $0, \infty$ & 0,00 & 0,00 & 1,00 & 0,00 & 0,40 & 0,00 & 0,00 & 0,50 & 0,00 \\
\hline Microorg an ism & 0,07 & 0,08 & 0,09 & 0,11 & 0,00 & 0,00 & 1,00 & 0,00 & 0,00 & 0,00 & 0,00 & 0,00 \\
\hline Pulp Bleaching & 0,07 & 0,00 & $0, \infty$ & 0,00 & 0,13 & 0,40 & 0,00 & 1,00 & 0,00 & 0,00 & 0,67 & 0,00 \\
\hline White Rot Fungi & 0,15 & 0,18 & 0,33 & 0,00 & 0,00 & 0,00 & 0,00 & 0,00 & 1,00 & 0,00 & 0,00 & 0,00 \\
\hline b-Glucosid ase & 0,17 & 0,00 & $0, \infty$ & 0,00 & 0,00 & 0,00 & 0,00 & 0,00 & 0,00 & 1,00 & 0,00 & 0,00 \\
\hline Chlorine DioxideBleaching & 0,00 & 0,00 & $0, \infty$ & 0,00 & 0,00 & 0,50 & 0,00 & 0,67 & 0,00 & 0,00 & 1,00 & 0,00 \\
\hline Consortium of Bacteria & 0,18 & 0,00 & 0,10 & 0,00 & 0,00 & 0,00 & 0,00 & 0,00 & 0,00 & 0,00 & 0,00 & 1,00 \\
\hline Deinking & 0,00 & 0,00 & 0,00 & 0,22 & 0,00 & 0,00 & 0,00 & 0,00 & 0,00 & 1,00 & 0,00 & 0,00 \\
\hline Glucosyltransferase & 0,00 & 0,20 & $0, \infty$ & 0,00 & 0,00 & 0,00 & 0,00 & 0,00 & 0,00 & 0,00 & 0,00 & 0,00 \\
\hline Laccase & 0,08 & 0,09 & 0,10 & 0,00 & 0,00 & 0,00 & 0,00 & 0,00 & 0,00 & 0,00 & 0,00 & 0,00 \\
\hline Lipase & 0,00 & 0,00 & 0,10 & 0,00 & 0,00 & 0,00 & 0,00 & 0,00 & 0,00 & 0,00 & 0,00 & 0,00 \\
\hline Oxi dored uc tase & 0,08 & 0,09 & $0, \infty$ & 0,00 & 0,00 & 0,00 & 0,00 & 0,00 & 0,00 & 0,00 & 0,00 & 0,00 \\
\hline Peroxidase & 0,08 & 0 & 0,10 & 0,00 & 0,00 & 0,00 & 0,00 & 0,00 & 0,00 & 0,00 & 0,00 & 0,00 \\
\hline
\end{tabular}

A partir de la matriz normalizada se desarrollo un análisis estadístico de escalamiento multidimensional con el software SPSS 17.0, con el cual se puede obtener relaciones numéricas entre diferentes variables (matriz $18 \mathrm{x}$
18) y este se puede representar en dos dimensiones. El mapa de coocurrencia de palabras que se obtuvo a través del análisis de escalamiento multidimensional se presenta en la figura 4. 
Figura 4

Mapa tecnológico por coocurrencia de palabras

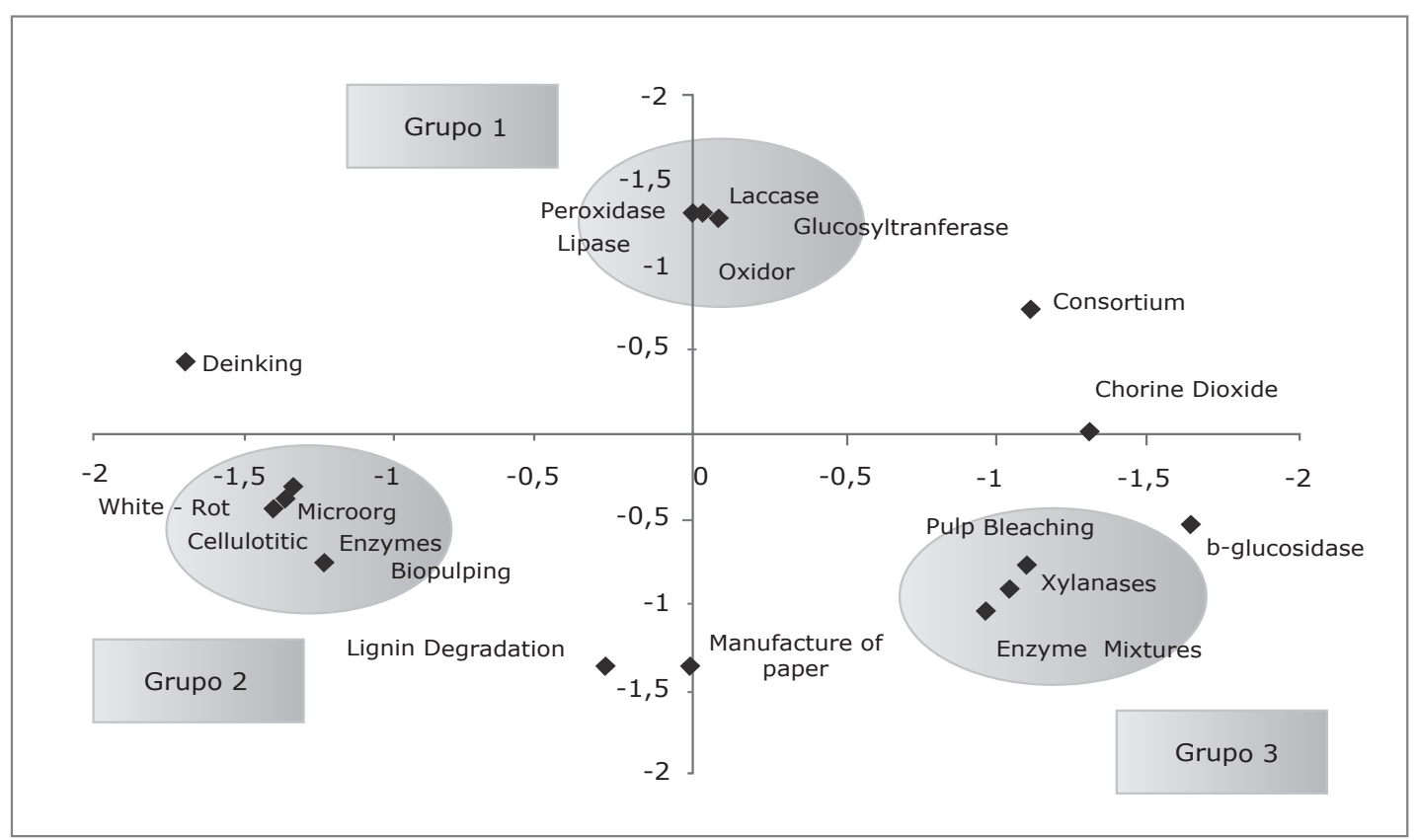

Interpretación del mapa tecnológico. Se ubicaron tres grupos de palabras como puede observarse en la figura 4.

En el grupo 1, se encuentra la utilización de actividades enzimáticas específicas, dichas palabras corresponden a patentes cuya innovación esta dada por un nuevo conjunto de componentes, que además del uso de la enzima incluyen aditivos $u$ otras sustancias para mejorar diferentes procesos de interés como la degradación de la lignina, el blanqueado de la pulpa, o bien nuevos procesos como la eliminación de tintas de impresoras laser para hacer pulpa desde papel reciclado, o el mejoramiento las cualidades del papel mediante la eliminación de compuestos que emitan fluorescencia. La generación de nuevo conocimiento sobre los procesos naturales especialmente en microbiología, a través de la investigación básica en biotecnología ha dado paso a la diversificación de las tecnologías emergentes, sin embargo aun son pocas en relación a desarrollos patentables de procesos industriales que se basan la transformación química tradicional.

En el grupo 2, se encuentran las palabras pertenecientes a desarrollos en los procesos de obtención de biopulpa, la diferencia entre dichas patentes consiste en el uso de diferentes 
microorganismos y/o sus componentes enzimáticos pero todos dirigidos al mismo proceso. Como se menciono anteriormente la Biodiversidad es uno de los pilares para el desarrollo de la biotecnología, se puede afirmar en el sentido biológico que es la totalidad de los genes, especies y ecosistemas de una región, representado en variedades vegetales, animales, fúngicas e incluso microbiológicas, modificadas tras siglos de selección para la acumulación de características deseables, esto último representa además diversidad en rutas metabólicas, lo que implica un amplio rango de posibilidades que recientemente se les explora aplicación industrial.

En el grupo 3. Se encuentran la combinación de tecnologías como el de enzimas junto con procesos de blanqueado diferentes a los enzimáticos, las mezclas de enzimas para procesos diferentes como la biopulpa y la degradación de lignina y la eliminación de tinta, que responden a diferentes procesos y necesidades relacionadas con la industria de la pulpa y el papel. Donde se observa un cambio en la concepción innovativa multicomponente de los procesos patentados, dado que las patentes que incluyeran desarrollo en biotecnología para la industria del papel y anteriores al año 2000, en general se dirigían solamente hacia el uso de la enzima lacassa para los procesos de bioblanqueado.

Los agrupamientos y las palabras dispersas en el mapa se puede interpretar como un área tecnológica en plena emergencia y que además presenta la posibilidad de enfocase desde puntos de vista y necesidades muy diferentes, relacionados con la manufactura del papel, como ya se discutió esto esta relacionado con el creciente y reciente conocimiento sobre el mundo microbiano y su inmensa diversidad no solo en términos de especies, sino aun mas diverso en rutas y procesos metabólicos que actualmente son temas relevancia en investigación básica y aplicada. En Colombia se han realizado varios estudios para el tratamiento de aguas residuales de la industria papelera a escala de laboratorio (Martínez et al.,2005) y existen grupos dedicados al estudio de la aplicabilidad de microorganismos y sus enzimas a la industria del papel, (Grupo de Biotecnología Ambiental e Industrial de la Universidad Javeriana, Grupo BIOALLI de la Universidad de Antioquia, Grupo de la Universidad EAFIT) pero aun el potencial biotecnológico de estos trabajos esta por explotar.

La biodiversidad es patrimonio de los países que lo poseen (Cumbre de la Tierra, Río de Janeiro 1992) explotada por los países desarrollados sin retribución, para el país a donde pertenecen tales recursos o para las comunidades indígenas poseedoras del conocimientos ancestrales. El valor que tienen sus recursos naturales no renovables y la biodiversidad esta dado en gran medida por el desarrollo de nuevos bioproductos e industrias usando los recursos genéticos de la flora y fauna, de microorganismos y de otros recursos biológicos, en esta medida el 
aprovechamiento de la biodiversidad mediante la biotecnología, permite desarrollos sostenibles con potencial de aumentar la productividad de restaurar y proteger el medio ambiente, lo que representa oportunidades para el país, sin embargo el desarrollo áreas de la biotecnología en Colombia esta limitado por problemas como la creciente brecha científica y tecnológica con respecto al de los países industrializados, además la ausencia de incorporación de la biotecnología plantea serias amenazas, dado que puede causar la perdida de la competitividad frente a los países que están aplicando innovaciones biotecnológicas en forma creciente (Torres, 2002). Este ejercicio constituye un primer paso a seguir en los procesos de Vigilancia Tecnológica que contribuyan a la definición de políticas y direccionamiento de la gestión tecnológica para que la cadena de papel y artes gráficas alcance mayor competitividad y mayor inserción en el mercado internacional globalizado.

\section{Conclusión}

A partir del mapa se puede interpretar la aplicación de desarrollos biotecnológicos como un área tecnológica en plena emergencia, que además presenta la posibilidad de enfocase desde puntos de vista y necesidades muy diferentes, relacionados con la manufactura del papel, tales desarrollos están relacionado con el creciente y reciente conocimiento sobre el mundo microbiano y su inmensa diversidad no solo en términos de especies, sino aun mas diverso en rutas y procesos metabólicos que actualmente son temas relevancia en investigación básica y aplicada. Sin embargo en Colombia la poca aplicación de dichos desarrollos hace que se pierda competitividad frente a los países industrializados.
Gorbaneff y colaboradores (2006) proponen que los ambientes en los cuales surgen las empresas biotecnológicas exitosas se deben a: el acceso a la tecnología. El acceso a los recursos financieros de alto riesgo. El desarrollo del talento humano, capaz de desenvolverse en el ambiente inestable, que se caracteriza por la "destrucción de las competencias" adquiridas previamente. La creación de los fuertes incentivos para el personal que trabaja en las empresas biotecnológicas innovadoras, como marco referencial del estado actual de la biotecnología en Colombia aplicable al contexto empresarial colombiano en el país se cuenta con una tradicional infraestructura de investigación en biotecnología y las 
aéreas relacionadas "El Valle, con 225 grupos de investigación de Colciencias, ocupa el tercer lugar en el país. Esta cifra corresponde al $10.1 \%$ del total de grupos en Colombia. El departamento es precedido por Bogotá con 871 grupos $(39.1 \%)$ y Antioquia con 370 grupos (16.6\%). En América Latina predomina que la mayor concentración de los proyectos biotecnológicos se genera en el sector académico (45\%), lo que puede interpretarse como el buen desempeño que los investigadores, pero también la falta de empresas de base tecnológica en el país. Esta situación, común a Colombia y a otros países de América Latina, demuestra como las regiones con un nivel de desarrollo relativamente bajo aprovechan sus recursos naturales pero no hay un valor agregado sustancial en ellos que permita tener un dinamismo creciente en los ingresos a partir de su demanda interna y/o de la exportación de dichos productos (CIECI, 2007). Para la generación de bionegocios se requiere iniciar un proceso de modernización del aparato productivo que conduzca a una reestructuración de la actividad industrial del departamento a partir del uso de los recursos biológicos existentes, que por una parte logre el posicionamiento de nuevas empresas con una alta incorporación de tecnología y conocimiento, siendo la biotecnología una de las herramientas de innovación con distintivos muy peculiares de competitividad global y generadoras de valor agregado tecnológico, ecológicamente sostenibles y por la otra un aumento considerable de fuentes de empleo cualificado. Lo que implica retos frente al nivel de desarrollo y apropiación tecnológica para el país en los próximos años, y adopción de políticas para optimizar la estructura de las empresas de biotecnología, con miras a contribuir a su mayor competitividad. 


\section{Bibliografía}

Biotechnology Center of Excellence Corporation. 2003. Posibilidades de la biotecnología para el uso sostenible de los recursos de biodiversidad en la región andina: recomendaciones y directrices estratégicas. Informe presentado a: Corporación Andina de Fomento (CAF) Caracas, Venezuela y a la Comisión Económica Para América Latina y El Caribe de Las Naciones Unidas de Las Naciones Unidas (CEPAL) Santiago, Chile.

Cámara de Comercio de Bogotá. 2005. Caracterización de Las Cadenas Productivas de Manufactura y Servicios en Bogotá Y Cundinamarca. Informe de Vicepresidencia de Gestión Cívica y Social Dirección de Estudios e Investigaciones Bogotá, D.C.

Cala-Hederich D.F. 2007. Los Doce Años de Corpodib. Revista Colombiana de Biotecnología. IX (1): 3-4.

Cárama de Comercio de Bogotá.2006.Balance tecnológico de la Cadena Productiva Hortofrutícola en Bogotá y Cundinamarca. Departamento de publicaciones.Bogotá D.C.

Castellanos O. del Portillo, P. de Peña, M. 2006. Análisis prospectivo de la biotecnología en Colombia 2005 - 2015 en el marco del direccionamiento estratégico., Revista Avances de pensamiento estratégico y prospectiva, febrero, No.2.

Castellanos, O.F. Ramírez, D.C. Montañez, V.M. 2006. Perspectiva en el desarrollo de las enzimas industriales a partir de la inteligencia tecnológica. Revista Ingeniería $E$ Investigación. 26(2): 52-67.

CIECI-Centro de investigaciones en Economía y competitividad internacional Pontificia Universidad Javeriana-Cali.2007. Plan de la Estrategia Bioregión Valle del Cauca 2019.

COLCIENCIAS-Instituto Colombiano para el Desarrollo de la Ciencia y la Tecnología "Francisco José de Caldas", 1999. Programa Nacional de Biotecnología. Plan Estratégico 1999-2004. Bogotá.

COLCIENCIAS - CORPOGEN. 2008. La Biotecnología Motor de Desarrollo para la Colombia de 2015.

CORPODIB. 2002. Bioetanol por fermentación del jugo de caña, de azúcar y melazas como aditivo oxigentante de la gasolina: Resumen del estudio realizado por Corpodib Kilborn SNC - Lavalin. Santafé de Bogotá. D.C. 
Corporación Biotec.2006. Corporación para El Desarrollo de La Biotecnología. Resumen Ejecutivo.

Correa H.D, Ruiz S.L. y Arévalo. L.M. (eds) 2005. Plan de acción en biodiversidad de la cuenca del Orinoco - Colombia / 2005 - 2015 - Propuesta Técnica. Bogotá D.C.: Corporinoquia, Cormacarena, I.A.v.H, Unitrópico, Fundación Omacha, Fundación Horizonte Verde, Universidad Javeriana, Unillanos, WWF - Colombia, GTZ - Colombia.

Departamento Nacional de Planeación 2007. Agenda Interna para la Productividad y la Competitividad. Documento sectorial, Cadena pulpa, papel, cartón, Industria Gráfica, Industria Editorial. Bogotá, D. C.

González G. Castellanos, O. 2000. Incidencia de los mercados en el desarrollo de la ingeniería de enzimas en Colombia., Ingeniería y Sociedad, 6, 2000.

Gorbaneff, y AArón, I.C., Chávez, C. 2006. Restricciones Estructurales Para La Innovación en La Biotecnología Colombiana. Cuadernos de Administración (Colombia). 19 (31): 103-143.

Hao J. Song, F. Huang, Yang, C. Zhang, Z. Zheng, Y. Tian, X. 2007. Production of laccase by a newly isolated deuteromycete fungus Pestalotiopsis sp. and its decolorization of azo dye. J Ind Microbiol Biotechnol. 34:233-240.

Ivchenko, G.I. Honov, S.A. 1998. On the Jaccar`s similarity test. Journal of Mathematical Sciences, Vol. 88, No. 6.

Kar, S. Mandal, A. Das Mohapatra, P.K. Mondal, K.C. Pati, B.R. 2006. Production of Cellulase-Free Xylanase by Trichoderma Reesei SAF3. Brazilian Journal of Microbiology. 37:462-464.

León Am, Catellanos OF,Vargas FA. 2006. Valoración, selección y pertinencia de herramientas de software utilizadas en vigilancia tecnológica. Ing Investig, 26(1).

Lucero Mafla, A.M. Peña Villamil, L.A. Bacca Ibarra, T. 2004. Evaluación de la actividad biocontroladora de Beauveria bassiana y Metarhizium anisopliaesobre larvas de Ancognatha scarabaeiodes (Coleoptera:Scarabaeidae) Revista Corpoica. 51(1): 43-48.

Martínez-Salgado M. Pedrosa-Rodríguez A. Rodríguez-Vázquez, R. Rosas-Acosta J. 2005. Efecto De La Glucosa y Nitrato de Amonio Sobre Las Enzimas Ligninolíticas Producidas Por Trametes versicolor Inmovilizado en Espuma y La Decoloración de Un Efluente Papelero En Un Biorreactor de Lecho Fluidizado. Universitas Scientiarum. 10(2): 27-36. 
Monsalve G, J.F. Medina de Pérez, V.I. Ruiz Colorado, A. 2006. Producción de Etanol a Partir de la Cáscara de Banano y de Almidón de Yuca. DYNA. 73 (150): 21-27.

Olaya, D.L. y Orozco, L. 2004. Indicadores del Programa Nacional de Biotecnología. Observatorio de Ciencia y Tecnología, Bogotá.

Roca, W. 2004. Estudio de las Capacidades Biotecnológicas e Institucionales para el Aprovechamiento de la Biodiversidad de los Países de la Región Andina. Informe preparado para la Comisión Económica para América Latina y el Caribe (CEPAL) y la Corporación Andina de Fomento. Centro Internacional de la Papa (CIP) Lima-Perú.

Sapre, H.J. Patil, M.B. 2005. Purification and characterization of a thermoalkalophilic xylanase from Bacillus sp. World Journal of Microbiology \& Biotechnology. 21:649-654

Torres, R. 2002. Bases para una política nacional de biotecnología. Informe final presentado al Departamento Nacional de Planeación - DNP. Dirección de Desarrollo Agrario, Bogotá D.C.

Vargas, F. Y Castellanos, O. 2005. Vigilancia como herramienta de innovación y desarrollo tecnológico. Caso de aplicación: Sector de empaques plásticos flexibles. Revista Ingeniería e Investigación. 25(2): 32-41. 